\title{
Utilización del extracto oleoso de Pleuroncodes monodon como pigmentante en el alimento balanceado de la trucha arcoíris (Oncorhynchus mykiss)
}

\author{
Use of oily extract of Pleuroncodes monodon as pigment in the feed of the \\ rainbow trout (Oncorhynchus mykiss)
}

\author{
Paul Montufar ${ }^{1}$, Marisol Churacutipa Mamani', \\ Maritza Barriga-Sánchez ${ }^{2,3}$
}

\section{Resumen}

\begin{abstract}
El objetivo del presente trabajo fue evaluar el extracto oleoso de la munida Pleuroncodes monodon en el alimento balanceado sobre la pigmentación del músculo de la trucha arcoíris (Oncorhynchus mykiss). Los tratamientos fueron T1: alimento formulado sin pigmento, T2 y T3: alimento con 70 y $90 \mathrm{mg}$ de astaxantina natural $/ \mathrm{kg}$ de alimento, respectivamente y, T4 alimento con pigmento sintético (70 $\mathrm{mg}$ de astaxantina/ $\mathrm{kg}$ de alimento). Se registraron cada 15 días y durante 90 días los valores de color utilizando la regla colorimétrica de Roche SalmoFan ${ }^{\mathrm{R}}$ y los valores de $\mathrm{L}^{*}, \mathrm{a}^{*}, \mathrm{~b}^{*}, \mathrm{C}^{*}$ y $\mathrm{H}^{*}$ de la zona dorsal y caudal de la trucha. Los resultados de las lecturas de color indicaron que los tratamientos T2, T3 y T4 obtenidos con la regla SalmoFan ${ }^{\mathrm{R}}$ no presentaron diferencias significativas entre ellas; no obstante, la medición obtenida con el colorímetro muestra diferencias de color del T4 con respecto al T2 y T3. Se concluye, que la adición de $70 \mathrm{mg}$ de astaxantina natural por kilogramo de alimento logra la pigmentación similar a lo obtenido con la astaxantina sintética.
\end{abstract}

Palabras clave: trucha arcoíris, pigmentación, astaxantina natural, Pleuroncodes monodon

\footnotetext{
${ }^{1}$ Truchas Arapa Perú SAC, Puno, Perú

${ }^{2}$ Laboratorio de Compuestos Bioactivos DIDITT, Instituto Tecnológico de la Producción, Callao, Perú

${ }^{3}$ E-mail: mbarriga@itp.gob.pe
}

Recibido: 13 de mayo de 2021

Aceptado para publicación: 10 de noviembre de 2021

Publicado: 22 de diciembre de 2021

CLos autores. Este artículo es publicado por la Rev Inv Vet Perú de la Facultad de Medicina Veterinaria, Universidad Nacional Mayor de San Marcos. Este es un artículo de acceso abierto, distribuido bajo los términos de la licencia Creative Commons Atribución 4.0 Internacional (CC BY 4.0) [https:// creativecommons.org/licenses/by/4.0/deed.es] que permite el uso, distribución y reproducción en cualquier medio, siempre que la obra original sea debidamente citada de su fuente original 
The IM of this study to evaluate the oily extract of the squat lobster Pleuroncodes monodon in the balanced feed on the pigmentation of the muscle of the rainbow trout (Oncorhynchus mykiss). The treatments were T1: feed formulated without pigment, T2 and T3: feed with 70 and $90 \mathrm{mg}$ of natural astaxanthin/ $\mathrm{kg}$ of feed, respectively, and T4 feed with synthetic pigment ( $70 \mathrm{mg}$ of astaxanthin $/ \mathrm{kg}$ of feed). The color values were recorded every 15 days and for 90 days using the Roche SalmoFan colorimetric rule and the $\mathrm{L}^{*}, \mathrm{a}^{*}, \mathrm{~b}^{*}, \mathrm{C}^{*}$ and $\mathrm{H}^{*}$ values of the dorsal and caudal zone of the trout. The results of the color readings indicated that the treatments T2, T3 and T4 obtained with the SalmoFan rule did not show significant differences between them; however, the measurement obtained with the colorimeter showed differences in the color of $\mathrm{T} 4$ with respect to T2 and T3. It is concluded that the addition of $70 \mathrm{mg}$ of natural astaxanthin per kilogram of feed achieves pigmentation similar to that obtained with synthetic astaxanthin.

Key words: rainbow trout, pigmentation, natural astaxanthin, Pleuroncodes monodon

\section{INTRODUCCIÓN}

En la trucha arcoíris (Oncorhynchus mykiss), los filetes con pigmentación roja se consideran mucho más deseables que los filetes blancos no pigmentados (Folkstead et al., 2008). Se conoce que los peces, al igual que otros animales, no pueden sintetizar sus propios pigmentos colorantes y deben obtenerlos de su dieta. En consecuencia, la pigmentación de los salmónidos de cultivo se ha logrado con la inclusión de carotenoides sintéticos ( $\beta$-caroteno, cantaxantina, zeaxantina y astaxantina) o fuentes naturales (levadura, bacterias, algas, plantas superiores y harina de crustáceos) en sus dietas (Shahidi y Brown, 1998). Sin embargo, casi todos los suplementos de pigmentos carotenoides en la alimentación del salmón y la trucha proceden de Carophyll Pink ${ }^{\mathrm{TM}}$ (Rahman et al., 2016). En ese sentido, el presente estudio propone evaluar nuevas fuentes de pigmentos naturales, para no continuar con la dependencia de los productos importados que enfrenta la truchicultura en el Perú.
Una alternativa es utilizar la munida (Pleuroncodes monodon), crustáceo del mar peruano que contiene astaxantina. Este artrópodo es usualmente capturado como pesca incidental de la anchoveta. El Instituto del Mar del Perú reportó 2201702 y 1687004 toneladas de biomasa de munida en verano y primavera, respectivamente (Castillo et al., 2020), evidenciando la gran cantidad de este recurso hidrobiológico en el mar peruano. El objetivo del presente estudio fue utilizar el extracto oleoso de la munida Pleuroncodes monodon en el alimento balanceado de trucha arcoíris (Oncorhynchus mykiss) y evaluar la pigmentación del músculo de trucha.

\section{Materiales y Métodos}

\section{Localización del Estudio}

El estudio fue realizado durante 90 días entre enero y abril de 2020 en el lago de Arapa, en la provincia de Azángaro, departamento de Puno, Perú, a una altitud de 3820 msnm. 
La temperatura del agua tuvo una media de $16.7^{\circ} \mathrm{C}$ y el oxígeno disuelto tuvo una media de $6.08 \mathrm{mg} / \mathrm{l}$ durante el desarrollo del experimento.

\section{Peces, Jaula, Dieta}

Peces

Se seleccionaron truchas arcoíris hembras de cuatro meses de edad aproximada de la línea Troutlodge. Los peces tenían de $35.71 \mathrm{~g}$ de peso promedio vivo y no habían recibido pigmentos en la dieta. Se trabajó con 512 peces, distribuidas al azar en 16 microjaulas (32 peces por jaula) (Figura 1 ).

\begin{tabular}{|c|c|c|c|c|c|}
\hline T1 & T4 & T3 & T1 & T3 & $\mathrm{T} 1$ \\
\hline T2 & & $\mathrm{T} 1$ & T3 & & T3 \\
\hline $\mathrm{T} 2$ & $\mathrm{~T} 2$ & T4 & T4 & $\mathrm{T} 2$ & T4 \\
\hline
\end{tabular}

Figura 1. Distribución de las microjaulas según tratamientos y repeticiones

\section{Jaulas}

Las microjaulas estaban sostenidas en dos jaulas flotantes construidas de forma artesanal $\left(5 \times 5 \mathrm{~m}^{2}\right)$, formando una estructura cuadrada, estando unidos a boyas y cilindros de plástico. Cada jaula flotante fue dividida en nueve áreas de las cuales se utilizaron ocho áreas, quedando la central vacía. Cada microjaula, construida con redes, tenía dimensiones de $1.6 \times 1.6 \times 2.1 \mathrm{~m}$ de largo, ancho y profundidad, respectivamente. Los peces se distribuyeron en cuatro tratamientos con cuatro repeticiones (Figura 1).

\section{Dieta de la trucha}

La munida Pleuroncodes monodon fue obtenida fresca en el muelle de Pisco y se transportó con hielo en una caja isotérmica a las instalaciones del Instituto Tecnológico de la Producción (ITP) en Lima, Perú, donde se realizó la deshidratación del crustáceo. El extracto oleoso (EO) se obtuvo con el equipo de extracción multisolvente (modelo 2802.0000, Top Industrie, Francia), equipado con una bomba de alta presión (90-2491 REV L, SSI), un chiller (PCPR 13.02-NED, National Lab), reactor (ø $163 \times 353 \mathrm{~mm})$ y tres separadores de acero (ø $78 \times 278 \mathrm{~mm}$ ). La temperatura de extracción y el flujo del $\mathrm{CO}_{2}$ así como del etanol fue controlada con el software del equipo, pero la presión fue controlada manualmente con una válvula reguladora de la presión. Se realizaron las extracciones con una técnica amigable al medio ambiente, conocida como técnica supercrítica. Se empleó el $\mathrm{CO}_{2}$ supercrítico + etanol. Los parámetros de extracción fueron flujo de $\mathrm{CO}_{2}$ de $40 \mathrm{~g} / \mathrm{ml}, 149 \mathrm{bar}, 48.5^{\circ} \mathrm{C}$ y $13 \%$ etanol, parámetros que fueron obtenidos mediante una optimización con el método superficie de respuesta (en proceso de patente en el INDECOPI), luego se evaporó el etanol en el rotavapor (Laborota 4003, Heidolph) a $40{ }^{\circ} \mathrm{C}$ quedando el EO de munida, el cual fue llevado a $-20 \pm 1{ }^{\circ} \mathrm{C}$ hasta su posterior análisis.

La astaxantina (Astx) del EO fue cuantificada siguiendo el método descrito por Sánchez-Camargo et al. (2011). Para ello, se preparó una solución de Astx (98.6\%, Dr Ehrenstofer) de $250 \mu \mathrm{g} / \mathrm{ml}$, a partir de la cual se preparó la curva estándar de Astx con cinco soluciones a concentraciones entre 1 a 5 $\mu \mathrm{g} / \mathrm{ml}$ con hexano. Se realizó la lectura de la absorbancia de la curva estándar y la muestra en un espectrofotómetro (UV-200 S, Shimadzu, Japón) a una longitud de onda de $472 \mathrm{~nm}$. Los carotenoides se expresaron en mg de Astx/kg de EO. Este método también 
Cuadro 1. Formulación del alimento T1

\begin{tabular}{lc}
\hline Ingredientes & $\begin{array}{c}\text { Mezcla } \\
(\%)\end{array}$ \\
\hline Harina de pescado & 31.0 \\
Harina de pota & 10.3 \\
Soya integral extruida & 25.0 \\
Harinilla de trigo & 8.0 \\
Harina de maíz & 5.1 \\
Aceite de pescado & 11.3 \\
Aceite de soya & 7.5 \\
Aminoácido Licina HCL & 0.29 \\
Aminoácido Metionina 99 & 0.5 \\
Aminoácido Treonina 98 & 0.11 \\
Mezcla premix acuicultura & 0.2 \\
DSM & 0.1 \\
Cloruro de colina & 0.1 \\
Harina de orégano & 0.4 \\
Arcilla montchack & 99.9 \\
\hline Total
\end{tabular}

fue utilizado para la determinación de la Astx en el músculo dorsal de la trucha cada 15 días, previa extracción de la grasa, expresándose los resultados en mg de Astx/kg.

En las instalaciones del laboratorio de experimentos de la empresa Truchas Arapa Perú SAC se realizó la inclusión del EO de la munida por aspersión a los pellets. Para ello se utilizó el EO (11 $000 \mathrm{mg}$ Astx/kg) y según los cálculos se adicionó la cantidad necesaria para lograr un alimento con 70 y $90 \mathrm{mg}$ de Astx por kilogramo de alimento. Además, se adquirió un lote de $10 \mathrm{~kg}$ de alimento comercial (Nicovita 150 P) que contenía Astx sintética.

Los tratamientos fueron $\mathrm{T} 1$ : alimento formulado (Cuadro 1) sin pigmento; T2 y T3: Alimento formulado con 70 y $90 \mathrm{mg}$ de Astx, respectivamente, y T4: Alimento comercial con pigmento sintético (Nicovita 150P; $70 \mathrm{mg}$ de Astx/kg de alimento). Las características nutricionales del alimento formulado y comercial se observan en el Cuadro 2. Se planteó un grupo testigo (T1) para comparar la talla y peso de las truchas que no recibieron Astx con aquellas truchas que recibieron Astx natural (T2 y T3) y Astx sintetica (T4).

Los peces fueron alimentados una vez al día, al voleo, después de las 07:00. Se hizo el registro de la talla y el peso con un ictiómetro y una balanza, respectivamente, de 10 peces al azar de cada microjaula a los 90 días.

\section{Evaluación de Color y Astx de la Trucha}

Cada 15 días fueron sacrificados dos especímenes por microjaula para realizar la medición de color. Los peces fueron llevados a las instalaciones de la planta de la empresa Truchas Arapa Perú S.A.C., en la sala de eviscerado se realizó el corte tipo mariposa (corte por encima del lomo del pez), el lavado y el retiro de las vísceras. En una caja de evaluación de color con iluminación de luz constante se realizó la comparación de la escala de color con la regla colorimétrica Roche SalmoFan ${ }^{\mathrm{R}}$, que va desde 20 (rojo claro) a 34 (salmonado oscuro). La muestra evaluada fue el músculo de la zona dorsal y caudal de la trucha. Las mediciones se hicieron por triplicado.

Paralelamente se realizó la medición del color de la muestra en la celda del colorímetro (Konica Minolta CR-5, Japón), utilizando la fuente luminosa D65. Se registraron las lecturas de $\mathrm{L}^{*}, \mathrm{a}^{*}, \mathrm{~b}^{*}, \mathrm{C}^{*}$ (croma) y $\mathrm{H}^{*}$ (tono) por triplicado.

Los especímenes fueron congelados y enviados a las instalaciones del ITP en Lima. Se descongelaron, se cortaron trozos de la zona dorsal y caudal y se trituraron por separado. 
Cuadro 2. Información nutricional del alimento formulado (T1) y comercial (T2, T3 y T4)

\begin{tabular}{lcccc}
\hline Componentes & $\begin{array}{c}\text { Formulado } \\
\text { (T1) }\end{array}$ & $\begin{array}{c}\text { Nicovita } 150 \\
\text { (T2) }\end{array}$ & $\begin{array}{c}\text { Nicovita } 150^{1} \\
\text { (T3) }\end{array}$ & $\begin{array}{c}\text { Nicovita 150P } \\
\text { (T4) }\end{array}$ \\
\hline Proteína (\%) & 44.74 & 40 & 40 & 40 \\
Grasa (\%) & 16.52 & 15 & 15 & 15 \\
Fibra (\%) & 1.10 & 3 & 3 & 3 \\
Ceniza (\%) & 14 & 12 & 12 & 12 \\
Humedad (\%) & 6.08 & 12 & 12 & 12 \\
Calibre & 6 & 6 & 6 & 6 \\
Astx (mg/kg) & 0 & $70^{*}$ & $90^{*}$ & 70 \\
\hline
\end{tabular}

*Adición en el alimento de astaxantina (Astx) de munida (Pleuroncodes monodon)

${ }^{1}$ Alimento balanceado para peces de 150 a $500 \mathrm{~g}$ de peso

\section{Análisis Estadístico}

Se empleó el programa Minitab v. 17 para realizar el análisis de varianza y la prueba de comparaciones múltiples de Tuckey con el nivel de significancia de $\alpha=0.05$.

\section{Resultados y Discusión}

\section{Talla y Peso de las Truchas}

Los mayores valores de talla y peso correspondieron a $\mathrm{T} 2$ y $\mathrm{T} 4$, aunque los tratamientos $\mathrm{T} 1$ y $\mathrm{T} 3$ presentaron altos valores de desviación estándar, pudiendo indicar que la adición de EO de munida no influyó negativamente en el peso o talla de los especímenes de trucha.

\section{Color en el Músculo}

Medición con la Regla Colorimétrica Roche SalmoFan ${ }^{\mathrm{R}}$

Los resultados de color de la zona dorsal y caudal (Cuadro 4) de T2 y T3 no presentaron diferencia significativa con respecto al T4 ( $p>0.05)$. Se llegaron a obtener valo-
Cuadro 3. Talla y peso promedio de truchas arcoíris a los 90 días de suministro de dietas con pigmentos

\begin{tabular}{ccc}
\hline & Peso $(\mathrm{g})$ & Talla $(\mathrm{cm})$ \\
\hline T1 & $362.7 \pm 34.2^{\mathrm{b}}$ & $28.6 \pm 1.3^{\mathrm{b}}$ \\
T2 & $386.3 \pm 18.4^{\mathrm{a}}$ & $29.6 \pm 1.5^{\mathrm{a}}$ \\
T3 & $346.4 \pm 31.9^{\mathrm{b}}$ & $29.1 \pm 1.4^{\mathrm{ab}}$ \\
T4 & $387.9 \pm 20.3^{\mathrm{a}}$ & $30.0 \pm 1.8^{\mathrm{a}}$ \\
\hline
\end{tabular}

T1: alimento formulado sin pigmento; $\mathrm{T} 2$ y T3: Alimento formulado con 70 y $90 \mathrm{mg}$ de Ast $/ \mathrm{kg}$, respectivamente, T4: Alimento comercial con pigmento sintético

res promedio superiores de color en la zona caudal en comparación con la zona dorsal. Resultados similares fueron reportados por No y Storebakken (1991). Se evidencia que la aplicación de la Astx natural de la munida y la Astx sintética logran una pigmentación similar en el músculo de la trucha.

Se encontró que la coloración de la zona dorsal fue similar a lo reportado por Enaro (2019) utilizando concentraciones de 75 y 90 
Cuadro 4. Valores de las tonalidades ${ }^{1}$ de la zona caudal y dorsal del músculo de trucha arcoíris obtenidos con la regla SalmoFan ${ }^{\mathrm{R}}$

\begin{tabular}{cccccccc}
\hline & & 15 días & 30 días & 45 días & 60 días & 75 días & 90 días \\
\hline Zona & T2 & $<20$ & $21.88 \pm 0.48^{\mathrm{a}}$ & $25.75 \pm 0.65^{\mathrm{a}}$ & $27.63 \pm 1.11^{\mathrm{a}}$ & $29.63 \pm 0.95^{\mathrm{a}}$ & $32.13 \pm 0.25^{\mathrm{a}}$ \\
caudal & T3 & $<20$ & $21.25 \pm 0.96^{\mathrm{a}}$ & $24.88 \pm 0.25^{\mathrm{a}}$ & $27.75 \pm 0.65^{\mathrm{a}}$ & $29.38 \pm 0.48^{\mathrm{a}}$ & $31.75 \pm 0.87^{\mathrm{a}}$ \\
& T4 & $<20$ & $21.5 \pm 0.41^{\mathrm{a}}$ & $25.50 \pm 0.41^{\mathrm{a}}$ & $28.25 \pm 0.65^{\mathrm{a}}$ & $30.00 \pm 0.71^{\mathrm{a}}$ & $32.38 \pm 0.48^{\mathrm{a}}$ \\
Zona & T2 & $<20$ & $20.75 \pm 0.29^{\mathrm{a}}$ & $22.25 \pm 0.65^{\mathrm{b}}$ & $24.75 \pm 0.50^{\mathrm{b}}$ & $26.88 \pm 0.85^{\mathrm{b}}$ & $28.38 \pm 0.75^{\mathrm{b}}$ \\
dorsal & T3 & $<20$ & $20.63 \pm 0.48^{\mathrm{a}}$ & $21.88 \pm 0.63^{\mathrm{b}}$ & $23.88 \pm 0.25^{\mathrm{c}}$ & $26.75 \pm 0.87^{\mathrm{b}}$ & $28.5 \pm 0.58^{\mathrm{b}}$ \\
& T4 & $<20$ & $20.63 \pm 0.48^{\mathrm{a}}$ & $22.38 \pm 0.75^{\mathrm{b}}$ & $24.88 \pm 0.25^{\mathrm{b}}$ & $26.88 \pm 0.75^{\mathrm{b}}$ & $29.0 \pm 0.41^{\mathrm{b}}$ \\
\hline
\end{tabular}

Los valores son expresados como la media \pm desviación estándar. Letras diferentes en cada columna indica que existen diferencias significativas $(p<0.05)$

${ }^{1}$ Desde 20 (rojo claro) a 34 (salmonado oscuro)

ppm de Astx sintética durante 95 días y con una frecuencia alimentaria de cuatro raciones diarias, quienes obtuvieron valores de 29 con la regla Roche SalmoFan ${ }^{\mathrm{R}}$. En otro estudio, Lázaro (2014) consiguió grados de 25 y 28 de la escala de color (3 dietas diarias de 90 ppm de Astx sintética) durante un periodo de alimentación de 30 y 45 días respectivamente; valor superior a lo encontrado en el presente trabajo en la zona caudal y dorsal. Esta variación podría deberse a la mayor cantidad de raciones con Astx recibidas cada día.

Eguía (2017) comparó Nicovita (70 ppm Astx sintética) con Naltech (66 ppm Astx sintética) encontrando valores medios de 24.81 y 24.66 , respectivamente, en un periodo de 80 días; mientras que Yesilayer (2020) determinó un color de 28.33 para truchas alimentadas con $50 \mathrm{mg}$ Astx sintética $/ \mathrm{kg}$, durante un periodo de tres meses, muy similar al obtenido en el presente estudio en la dorsal de los tratamientos T2 y T3.

\section{Medición con el colorimetro}

Con el transcurso del tiempo se evidenció que los valores $\mathrm{a}^{*} \mathrm{y}^{*}$ de los tratamientos T2, T3 y T4 incrementaron y los valores de L*y H* disminuyeron. El valor b* aumentó en un inicio y luego disminuyó (Cuadro 5). Similar comportamiento de los parámetros de color de la trucha alimentada con extractos de ají rojo (Capsicum annuum) fue mencionado por Arredondo-Figueroa et al. (2007). Con relación a los parámetros de medición de color, Wrolstad et al. (2005) afirman que el sistema de color L*, a* y b* no proporciona una definición precisa de color, ya que el color es tridimensional, y sugieren mediciones de $\mathrm{L}^{*}, \mathrm{C}^{*}$ y $\mathrm{H}^{*}$.

Torres et al. (2020), realizaron la extracción de carotenoides con aceite de girasol a partir del polen de abeja para la pigmentación de truchas arcoíris (O. mykiss) con peso inicial de $206.3 \pm 6.2 \mathrm{~g}$. Ellos plantearon cuatro dietas: testigo (sin pigmento), un control positivo con carotenoide comercial $50 \mathrm{mg}$ $\mathrm{kg}^{-1}$ (Lucantin ${ }^{\circledR}$ Pink); P1: aceite vegetal con $25 \mathrm{mg}$ carotenoides totales $\mathrm{kg}^{-1}$ y P2: aceite vegetal con $50 \mathrm{mg}$ de carotenoides totales $\mathrm{kg}^{-1}$ ) encontrando a las 8 semanas que los valores de $\mathrm{L}^{*}, \mathrm{a}^{*} \mathrm{y} \mathrm{b}^{*}$ para $\mathrm{P} 1$ fueron de $51.67 \pm 3.28,0.57 \pm 0.39$ y $5.00 \pm 0.93$ y para $\mathrm{P} 2$ de $51.58 \pm 1.86,1.10 \pm 0.56$ y $7.37 \pm 1.18$, respectivamente. Solo el valor $L^{*}$ del T2 de la zona caudal obtenido en el presente trabajo a 
Cuadro 5. Valores de los parámetros de color $\mathrm{L}^{*}, \mathrm{a}^{*}, \mathrm{~b}^{*}, \mathrm{C}^{*}$ y $\mathrm{H}^{*}$ del musculo de trucha arcoíris de la zona dorsal y caudal, determinado cada 15 días de iniciada la dieta con pigmentos

\begin{tabular}{|c|c|c|c|c|c|c|c|}
\hline & \multirow{2}{*}{$\begin{array}{c}\text { Tiempo } \\
\text { (días) }\end{array}$} & \multicolumn{3}{|c|}{ Zona dorsal } & \multicolumn{3}{|c|}{ Zona caudal } \\
\hline & & $\mathrm{T} 2$ & T3 & $\mathrm{T} 4$ & $\mathrm{~T} 2$ & T3 & $\mathrm{T} 4$ \\
\hline \multirow[t]{7}{*}{$\mathrm{L}^{*}$} & 0 & $62.01 \pm 0.05$ & $62.01 \pm 0.05$ & $62.01 \pm 0.05$ & $61.88 \pm 0.32$ & $61.88 \pm 0.32$ & $61.88 \pm 0.32$ \\
\hline & 15 & $57.38 \pm 0.40^{\mathrm{b}}$ & $56.29 \pm 0.78^{b}$ & $58.76 \pm 2.11^{\mathrm{a}}$ & $56.83 \pm 0.65^{\mathrm{a}}$ & $54.26 \pm 1.05^{\mathrm{b}}$ & $57.56 \pm 4.11^{\mathrm{a}}$ \\
\hline & 30 & $56.44 \pm 0.86^{\mathrm{a}}$ & $55.70 \pm 0.75^{\mathrm{ab}}$ & $54.86 \pm 0.93^{b}$ & $56.58 \pm 0.73^{\mathrm{a}}$ & $55.12 \pm 0.38^{b}$ & $54.37 \pm 0.50^{\mathrm{c}}$ \\
\hline & 45 & $55.89 \pm 1.20^{\mathrm{ab}}$ & $56.89 \pm 1.45^{\mathrm{a}}$ & $54.87 \pm 2.21^{\mathrm{b}}$ & $52.92 \pm 0.81^{\mathrm{a}}$ & $52.85 \pm 1.45^{\mathrm{a}}$ & $51.57 \pm 0.87^{\mathrm{b}}$ \\
\hline & 60 & $54.92 \pm 1.81^{\mathrm{a}}$ & $55.48 \pm 1.61^{\mathrm{a}}$ & $53.53 \pm 2.4^{\mathrm{a}}$ & $51.48 \pm 1.89^{\mathrm{a}}$ & $49.58 \pm 1.89^{\mathrm{b}}$ & $49.29 \pm 1.35^{\mathrm{b}}$ \\
\hline & 75 & $51.57 \pm 1.80^{\mathrm{a}}$ & $51.15 \pm 2.14^{\mathrm{a}}$ & $51.42 \pm 1.09^{a}$ & $47.92 \pm 0.98^{\mathrm{a}}$ & $47.91 \pm 1.07^{\mathrm{a}}$ & $47.42 \pm 0.84^{\mathrm{a}}$ \\
\hline & 90 & $49.68 \pm 1.86^{\mathrm{b}}$ & $49.00 \pm 1.97^{\mathrm{b}}$ & $51.71 \pm 1.01^{\mathrm{a}}$ & $46.07 \pm 1.25^{\mathrm{a}}$ & $44.96 \pm 1.57^{\mathrm{a}}$ & $45.26 \pm 2.31^{\mathrm{a}}$ \\
\hline \multirow[t]{7}{*}{$a^{*}$} & 0 & $4.14 \pm 0.78$ & $4.14 \pm 0.78$ & $4.14 \pm 0.78$ & $5.33 \pm 0.45$ & $5.33 \pm 0.45$ & $5.33 \pm 0.45$ \\
\hline & 15 & $10.00 \pm 1.20^{\mathrm{a}}$ & $7.70 \pm 0.43^{b}$ & $6.03 \pm 1.51^{\mathrm{c}}$ & $9.94 \pm 1.28^{\mathrm{a}}$ & $7.73 \pm 0.47^{\mathrm{b}}$ & $10.25 \pm 0.97^{\mathrm{a}}$ \\
\hline & 30 & $13.54 \pm 0.82^{\mathrm{b}}$ & $10.07 \pm 1.02^{\mathrm{c}}$ & $15.01 \pm 0.59^{\mathrm{a}}$ & $13.54 \pm 0.82^{\mathrm{b}}$ & $10.01 \pm 1.05^{\mathrm{c}}$ & $14.94 \pm 0.64^{\mathrm{a}}$ \\
\hline & 45 & $19.85 \pm 1.44^{\mathrm{a}}$ & $18.28 \pm 0.98^{\mathrm{b}}$ & $21.13 \pm 1.82^{\mathrm{a}}$ & $22.18 \pm 1.28^{\mathrm{ab}}$ & $20.64 \pm 1.64^{b}$ & $23.66 \pm 2.06^{\mathrm{a}}$ \\
\hline & 60 & $20.65 \pm 1.87^{\mathrm{b}}$ & $21.87 \pm 2.78^{\mathrm{b}}$ & $24.68 \pm 2.49^{\mathrm{a}}$ & $23.49 \pm 1.00^{\mathrm{b}}$ & $24.79 \pm 1.90^{\mathrm{b}}$ & $26.75 \pm 2.19^{a}$ \\
\hline & 75 & $24.49 \pm 1.72^{\mathrm{ab}}$ & $23.71 \pm 1.49^{\mathrm{b}}$ & $25.61 \pm 2.28^{\mathrm{a}}$ & $26.08 \pm 1.13^{\mathrm{a}}$ & $24.55 \pm 2.87^{\mathrm{a}}$ & $26.25 \pm 1.54^{\mathrm{a}}$ \\
\hline & 90 & $24.79 \pm 2.07^{\mathrm{b}}$ & $24.02 \pm 1.57^{b}$ & $26.66 \pm 1.20^{\mathrm{a}}$ & $26.21 \pm 1.42^{b}$ & $25.49 \pm 1.06^{\mathrm{b}}$ & $27.36 \pm 0.82^{\mathrm{a}}$ \\
\hline \multirow[t]{7}{*}{$\mathrm{b}^{*}$} & 0 & $10.22 \pm 0.86$ & $10.22 \pm 0.86$ & $10.22 \pm 0.86$ & $10.38 \pm 1.00$ & $10.38 \pm 1.00$ & $10.38 \pm 1.00$ \\
\hline & 15 & $15.01 \pm 1.69^{\mathrm{a}}$ & $14.30 \pm 0.87^{\mathrm{a}}$ & $15.33 \pm 2.05^{\mathrm{a}}$ & $15.59 \pm 0.60^{\mathrm{a}}$ & $14.25 \pm 0.90^{\mathrm{b}}$ & $14.46 \pm 2.03^{\mathrm{ab}}$ \\
\hline & 30 & $18.45 \pm 0.42^{\mathrm{a}}$ & $15.75 \pm 0.58^{c}$ & $17.79 \pm 0.50^{\mathrm{b}}$ & $18.45 \pm 0.42^{\mathrm{a}}$ & $15.79 \pm 0.54^{\mathrm{c}}$ & $17.76 \pm 0.50^{\mathrm{b}}$ \\
\hline & 45 & $22.32 \pm 0.83^{\mathrm{a}}$ & $21.93 \pm 0.54^{\mathrm{a}}$ & $22.03 \pm 0.93^{\mathrm{a}}$ & $22.65 \pm 0.85^{\mathrm{a}}$ & $22.21 \pm 1.19^{a}$ & $22.54 \pm 1.04^{\mathrm{a}}$ \\
\hline & 60 & $22.33 \pm 1.37^{b}$ & $23.24 \pm 1.31^{\mathrm{ab}}$ & $23.63 \pm 0.96^{\mathrm{a}}$ & $22.98 \pm 0.76^{\mathrm{a}}$ & $23.18 \pm 1.12^{\mathrm{a}}$ & $23.65 \pm 0.85^{\mathrm{a}}$ \\
\hline & 75 & $23.11 \pm 1.21^{\mathrm{a}}$ & $22.97 \pm 2.00^{\mathrm{a}}$ & $22.98 \pm 1.32^{\mathrm{a}}$ & $22.48 \pm 0.69^{a}$ & $22.19 \pm 0.65^{a}$ & $22.43 \pm 1.47^{\mathrm{a}}$ \\
\hline & 90 & $22.50 \pm 1.51^{\mathrm{a}}$ & $22.03 \pm 1.08^{\mathrm{a}}$ & $23.07 \pm 2.59^{\mathrm{a}}$ & $22.09 \pm 1.19^{\mathrm{a}}$ & $22.00 \pm 0.89^{\mathrm{a}}$ & $22.81 \pm 1.69^{\mathrm{a}}$ \\
\hline \multirow[t]{7}{*}{$\mathrm{C}^{*}$} & 0 & $11.05 \pm 0.89$ & $11.05 \pm 0.89$ & $11.05 \pm 0.89$ & $11.67 \pm 0.97$ & $11.67 \pm 0.97$ & $11.67 \pm 0.97$ \\
\hline & 15 & $18.09 \pm 1.56^{\mathrm{a}}$ & $16.25 \pm 0.66^{\mathrm{b}}$ & $16.50 \pm 2.3^{2 \mathrm{a} b}$ & $18.51 \pm 1.18^{\mathrm{a}}$ & $16.22 \pm 0.68^{\mathrm{b}}$ & $17.75 \pm 2.04^{\mathrm{a}}$ \\
\hline & 30 & $22.89 \pm 0.79^{a}$ & $18.71 \pm 1.02 \mathrm{~b}$ & $23.28 \pm 0.75^{\mathrm{a}}$ & $22.89 \pm 0.79 \mathrm{a}$ & $18.70 \pm 1.01^{\mathrm{b}}$ & $23.20 \pm 0.78^{a}$ \\
\hline & 45 & $29.88 \pm 1.52^{\mathrm{ab}}$ & $28.55 \pm 0.95^{\mathrm{b}}$ & $30.54 \pm 1.83^{\mathrm{a}}$ & $31.72 \pm 1.24^{\mathrm{ab}}$ & $30.33 \pm 1.93^{b}$ & $32.68 \pm 2.18^{a}$ \\
\hline & 60 & $30.43 \pm 2.04^{b}$ & $31.94 \pm 2.76^{\mathrm{ab}}$ & $34.19 \pm 2.34^{\mathrm{a}}$ & $32.86 \pm 1.05^{\mathrm{b}}$ & $33.95 \pm 1.87^{\mathrm{b}}$ & $35.72 \pm 2.12^{\mathrm{a}}$ \\
\hline & 75 & $33.69 \pm 1.90^{\mathrm{a}}$ & $33.03 \pm 2.16^{\mathrm{a}}$ & $34.42 \pm 2.47^{\mathrm{a}}$ & $34.44 \pm 1.18^{\mathrm{a}}$ & $33.16 \pm 1.87^{\mathrm{a}}$ & $34.54 \pm 1.86^{\mathrm{a}}$ \\
\hline & 90 & $33.48 \pm 2.44^{\mathrm{ab}}$ & $32.60 \pm 1.80^{\mathrm{b}}$ & $35.30 \pm 2.15^{\mathrm{a}}$ & $34.29 \pm 1.73^{\mathrm{ab}}$ & $33.68 \pm 1.12^{\mathrm{b}}$ & $35.64 \pm 1.44^{\mathrm{a}}$ \\
\hline \multirow[t]{7}{*}{$\mathrm{H}^{*}$} & 0 & $67.95 \pm 3.97$ & $67.95 \pm 3.97$ & $67.95 \pm 3.97$ & $62.74 \pm 2.53$ & $62.74 \pm 2.53$ & $62.74 \pm 2.53$ \\
\hline & 15 & $56.21 \pm 4.68^{c}$ & $61.63 \pm 2.47^{\mathrm{b}}$ & $68.10 \pm 3.08^{\mathrm{a}}$ & $57.63 \pm 2.44^{\mathrm{b}}$ & $61.44 \pm 2.66^{\mathrm{a}}$ & $54.48 \pm 3.13^{\mathrm{c}}$ \\
\hline & 30 & $53.76 \pm 1.18^{\mathrm{b}}$ & $57.48 \pm 1.75^{\mathrm{a}}$ & $49.87 \pm 0.41^{\mathrm{c}}$ & $53.76 \pm 1.18^{\mathrm{b}}$ & $57.70 \pm 1.82^{\mathrm{a}}$ & $49.94 \pm 0.54^{\mathrm{c}}$ \\
\hline & 45 & $48.41 \pm 1.28^{b}$ & $50.22 \pm 1.22^{\mathrm{a}}$ & $46.27 \pm 1.72^{c}$ & $45.63 \pm 1.66^{\mathrm{b}}$ & $47.14 \pm 1.14^{\mathrm{a}}$ & $43.68 \pm 1.31^{\mathrm{c}}$ \\
\hline & 60 & $47.29 \pm 2.10^{\mathrm{a}}$ & $46.91 \pm 2.50^{\mathrm{a}}$ & $43.87 \pm 2.19^{\mathrm{b}}$ & $44.39 \pm 1.21^{\mathrm{a}}$ & $43.13 \pm 1.99^{\mathrm{ab}}$ & $41.56 \pm 1.64^{b}$ \\
\hline & 75 & $43.38 \pm 1.54^{\mathrm{ab}}$ & $44.05 \pm 2.07^{\mathrm{a}}$ & $41.96 \pm 1.48^{b}$ & $40.77 \pm 1.00^{\mathrm{a}}$ & $42.31 \pm 4.18^{\mathrm{a}}$ & $40.50 \pm 1.73^{\mathrm{a}}$ \\
\hline & 90 & $42.27 \pm 1.38^{\mathrm{a}}$ & $42.56 \pm 1.08^{a}$ & $40.76 \pm 2.99^{a}$ & $40.13 \pm 1.14^{\mathrm{a}}$ & $40.80 \pm 1.39^{a}$ & $39.77 \pm 1.89^{a}$ \\
\hline
\end{tabular}

Los valores son expresados como la media \pm desviación estándar. $\}$

Letras diferentes en cada fila para la zona dorsal indica que existen diferencias significativas $(p<0.05)$ Letras diferentes en cada fila para la zona caudal indica que existen diferencias significativas $(p<0.05)$ 

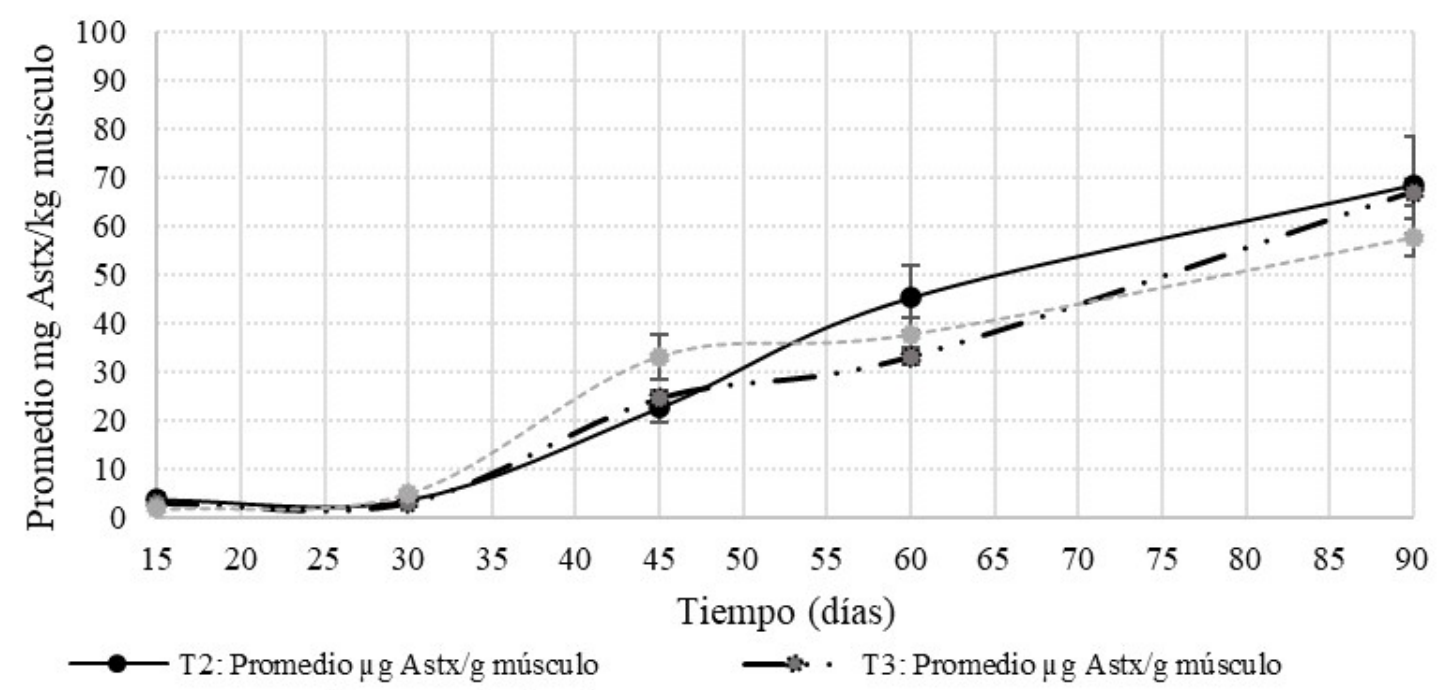

$\longrightarrow$ T3: Promedio $\mu \mathrm{g}$ Astx/g músculo

Figura 2. Valores de astaxantina (Astx) en $\mathrm{mg} / \mathrm{kg}$ de músculo de trucha arcoíris según la dieta con pigmentos

los 60 días (Cuadro 5) fue similar al obtenido en el estudio de Torres et al. (2020).

Así mismo, en un trabajo con cinco dietas experimentales $(0,15,30,60$ y $100 \%$ de proteína de harina de krill) en trucha arcoíris de peso inicial $102.06 \pm 0.04 \mathrm{~g}$ (Wei et al., 2019), se obtuvieron valores de $L^{*}$ similares a los obtenidos a los 90 días en la zona caudal de este trabajo; en tanto que Choubert et al. (2006), investigaron el efecto de dos aceites (oliva y pescado) sobre la eficacia pigmentante de la Astx (sintética y de microalgas verdes Haematococcus. pluvialis) en la trucha arcoíris con peso inicial de $185 \mathrm{~g}$ encontrando que los valores de $\mathrm{a}^{*}$ y $\mathrm{H}^{*}$ de las truchas que recibieron dietas durante seis semanas con aceite de pescado + Astx sintética $\left(80.2 \mathrm{mg} \mathrm{kg}^{-1}\right)$ y con aceite de oliva + Astx sintética $\left(78.1 \mathrm{mg} \mathrm{kg}^{-1}\right)$ fueron comparables con los obtenidos en este trabajo en el rango de 15 a 30 días de estudio.
Las diferencias de los valores de $\mathrm{L}^{*}$, $a^{*}, b^{*}, C^{*}$ y H* de este trabajo con los reportados por otros investigadores podrían deberse a que en este estudio se inició con truchas de peso promedio $35 \mathrm{~g}$ y los autores citados iniciaron la evaluación con truchas de pesos superiores.

\section{Astx en el Músculo}

El incremento del contenido de Astx en el músculo de trucha de los tratamientos T2, T3 y T4 fue muy bajo durante los primeros 30 días, aumentando a partir de dicho periodo (Figura 2).

T2 y T3 a los 45 días presentaron contenidos de Astx cercanos a los obtenidos por Martínez (2017), quien logró obtener una media de $23 \mathrm{mg}$ Astx/kg de músculo durante 10 meses de alimentación con Astx sintética, y a Saez et al. (2016), quienes obtuvieron un 
valor máximo de $24 \mathrm{mg}$ Astx/ $\mathrm{kg}$ a las $24 \mathrm{se}-$ manas. Por su parte, Pokniak et al. (2001) obtuvieron 20.5 y 15.4 ppm de Astx con dietas con concentraciones de 80 y 25 ppm de Astx sintética, respectivamente a los 60 días. Asimismo, Choubert et al. (2006) evidencian que los contenidos de Astx muscular en peces alimentados con Astx sintética son superiores a los alimentados con Astx de microalga (H. pluvialis).

\section{Conclusiones}

Las mediciones de color obtenidas con la regla SalmoFan ${ }^{\mathrm{R}}$ de las truchas alimentadas con 70 y $90 \mathrm{mg}$ astaxantina natural $/ \mathrm{kg}$ alimento a los 90 días no presentaron diferencia significativa con la trucha alimentada con astaxantina sintética $(\mathrm{p}>0.05)$. En forma similar, los valores promedio de $\mathrm{H}^{*}$ obtenidos con el colorímetro no presentaron diferencia significativa $(p>0.05)$ entre tratamientos.

\section{Agradecimientos}

Se agradece al Programa Nacional de Innovación en Pesca y Acuicultura, a la empresa Truchas Arapa Peru S.A.C. y al Instituto Tecnológico de la Producción por el financiamiento del proyecto PNIPA ACU SIADE 00017.

\section{Literatura Citada}

1. Arredondo-Figueroa JL, Ingle de la Mora G, Ponce-Palafox JT, BarrigaSosa I, Vernon-Carter EJ .2007. Color of raw, frozen, and smoked fillets of rainbow trout (Oncorhynchus mykiss) fed diets supplemented with astaxanthin and saponified red chilli (Capsicum annuum) extracts. J Aquat Food Prod T 16:35-50. doi: 10.1300/J030v16n01_04

2. Choubert G, Mendes-Pinto MM, Morais R. 2006. Pigmenting efficacy of astaxanthin fed to rainbow trout
Oncorhynchus mykiss: effect of dietary astaxanthin and lipid sources. Aquaculture 257: 429-436. doi: 10.1016/ j.aquaculture.2006.02.055

3. Eguía MJ. 2017. Influencia de dos marcas comerciales de alimento en el crecimiento y pigmentación muscular de la trucha (Oncorhynchus mykiss) en estanques. Tesis de Ingeniero Pesquero. Lima: Univ. Nacional Agraria La Molina. 50 p.

4. Enaro ED. 2019. Evaluación del desempeño productivo y grado de pigmentación de trucha arcoíris alimentadas con dos alimentos comerciales, durante el estadio de engorda. Tesis de Ingeniero Pesquero. Tacna: Univ. Nacional Jorge Basadre Grohmann. 122 p.

5. Folkstead A, Wold JP, Rorvik K, 2008. Rapid and non-invasive measurements of fat and pigment concentrations in live and slaughtered Atlantic salmon (Salmo salar L). Aquaculture 280: 129-135.

6. Castillo R, Cornejo R, La Cruz L, Grados D, Cuadros G, Valdez C, Pozada M. 2020. Distribución y biomasa de recursos pelágicos neríticos y oceánicos en el ecosistema marino peruano obtenidos de los cruceros hidroacústicos en el 2019. Bol Inst Mar Perú 35: 213- 241.

7. Lázaro R. 2014. Pigmentación de trucha arcoíris (Oncorhynchus mykiss) en fase de acabado, durante el almacenamiento en refrigeración y proceso de cocción, en el Centro Piscícola El Ingenio. Tesis de Ingeniero Zootecnista. Tingo María, Perú: Univ. Nacional Agraria de la Selva. 94 p.

8. Martínez MJ. 2017. Evaluación de dos pigmentos en la dieta de truchas arcoíris (Oncorhynchus mykiss) cultivada en centro de engorda. Tesis de Acuicultura. Puerto Montt, Chile: Univ. Austral de Chile. $44 \mathrm{p}$.

9. No HK, Storebakken T. 1991. Pigmentation of rainbow trout with astaxanthin at different water temperatures. Aquaculture 97: 203-216. doi: 10.1016/00448486(91)90265-9 
10. Pokniak J, Cornejo S, Bravo I, Battaglia J. 2001. Pigmentación de trucha arco iris (Oncorhynchus mykiss) tipo mar alimentadas con dos niveles de astaxantina en dietas de crecimientoengorda. Arch Med Vet 33: 155-164.

11. Rahman MM, Khosravi S, Chang K, Lee SM. 2016. Effects of dietary inclusion of astaxanthin on growth, muscle pigmentation and antioxidant capacity of juvenile rainbow trout (Oncorhynchus mykiss). Prev Nutr Food Sci 21: 281288. doi: 10.3746/pnf.2016.21.3.281

12. Sánchez-Camargo AP, Martinez-Correa HA, Paviani LC, Cabral FA. 2011. Supercritical $\mathrm{CO}_{2}$ extraction of lipids and astaxanthin from Brazilian redspotted shrimp waste (Farfantepenaeus paulensis). J Supercrit Fluid 56: 164-173. doi: 10.1016/j.supflu.2010.12.009

13. Shahidi F, Brown JA. 1998. Carotenoid pigments in seafoods and aquaculture. Crc Cr Rev Food Sci 38: 1-67. doi: 10.1080/10408699891274165

14. Torres EG, Fuenmayor CA, Vasquez SM, Díaz-Moreno C, Suárez H. 2020. Effect of bee pollen extract as a source of natural carotenoids on the growth performance and pigmentation of rainbow trout (Oncorhynchus mykiss). Aquaculture 514: 734490. doi: 10.1016/ j.aquaculture. 2019.734490

15. Saez PJ, Abdel Aal ESM, Bureau DP. 2016. Feeding increasing levels of corn gluten meal induces suboptimal muscle pigmentation of rainbow trout (Oncorhynchus mykiss). Aquac Res 47: 19721983. doi: 10.1111/are.12653

16. Wrolstad RE, Dursta RW, Jungmin Lee J. 2005. Tracking color and pigment changes in anthocyanin products. Trends Food Sci Tech 16: 423-428. doi: 10.1016/ j.tifs.2005.03.019

17. Yeilayer N. 2020. Comparison of flesh colour assessment methods for wild brown trout (Salmo trutta macrostigma), rarmed rainbow trout (Oncorhynchus mykiss) and farmed Atlantic salmon (Salmo salar). Pak J Zool 52: 10071014. doi: 10.17582/journal.pjz/20190520140524

18. Wei Y, Chen H, Jia M, Zhou H, Zhang $\boldsymbol{Y}, \boldsymbol{X u} \boldsymbol{W}$, Zhang W, Mai $\mathrm{K}$. Effects of dietary Antarctic krill Euphausia superba meal on growth performance and muscle quality of triploid rainbow trout Oncorhynchus mykiss farmed in sea water. Aquaculture 509: 72-84. doi: 10.1016/j.aquaculture.2019.05.013 